\title{
NONPROPORTIONAL LOADING EFFECTS ON ELASTIC-PLASTIC BEHAVIOR BASED ON STRESS RESULTANTS FOR THIN PLATES OF STRAIN HARDENING MATERIALS
}

\author{
C.H. ChOU, ${ }^{*}$ J. PAN,$\dagger$ and S.C. TANG $\ddagger$ \\ *Edison Welding Institute, $†$ The University of Michigan, and $\ddagger$ Ford Motor Company
}

\begin{abstract}
A stress resultant constitutive law in rate form is constructed for power-law hardening materials. The change of plate thickness is considered in the constitutive law. The elastic-plastic behavior of a plate element based on the stress resultant constitutive law under uniaxial combined tension and bending is determined under a limited number of nonproportional and unloading paths. The results based on the stress resultant constitutive law and the through-thethickness integration method are compared within the context of both the small-strain and finite deformation approaches. The results indicate that the selection of the normalized equivalent stress resultant and the corresponding work-conjugate normalized equivalent generalized strain is appropriate for describing the hardening behavior in the stress resultant space. However, the hardening rule in a power law form must be modified for low hardening materials at large plastic deformation when finite deformation effects are considered.
\end{abstract}

\section{INTRODUCTION}

Inelastic behavior of plate and shell structures has been analyzed by use of stress resultants in the last few decades (ILYUSHIN [1948], CRISFIELd [1974], BIENIEK \& Funaro [1976], Eggers \& KRöPLIN [1978], EIDSheIM \& LARSEN [1981], PAPAdopoulos \& TaYlor [1990]). In the analyses of plate and shell structures where a plate or shell element is plastically deformed, constitutive laws and flow rules based on stresses must be employed, and then, in general, stress resultants are determined from integrating stresses through the thickness. This method is referred to as the through-the-thickness integration method. The stress states through the thickness are usually complicated and dependent upon the entire deformation history of the plastically deformed plate or shell element. However, when a macroscopic viewpoint is adopted, stress resultant constitutive equations are employed and the local stress variation through the thickness then cannot be accounted for. Nevertheless, when the stress resultant theory is employed in structural analyses by finite element methods, one less dimension of calculation and less memory space are needed as compared with those using the through-the-thickness integration method.

From phenomenological viewpoints, perfectly plastic models or strain hardening models with a limit yield surface are assumed in those stress resultant constitutive laws mentioned above except in that of PAPADOPOulos and TAYLOR [1990] where linear strain hardening is assumed. In contrast, CHOU et al. [1991] derived a stress resultant constitutive law based on the Kirchhoff assumption and the $J_{2}$ deformation plasticity theory for power-law hardening materials. This constitutive law was developed under the 
assumptions of large plastic strains and nearly proportional loading paths. Under these assumptions, the elastic strains are neglected and the use of the $J_{2}$ deformation plasticity theory is justified (BUDIANSKY [1959]). In applications, however, loading paths cannot be guaranteed to be nearly proportional and a simple closed-formed yield condition is preferred. Therefore, CHOU et al. [1993] constructed a modified Ilyushin yield surface and derived a hardening rule in the stress resultant space. Then, an appropriate rate form of the elastic-plastic stress resultant constitutive law can be established based on the yield surface and the hardening rule.

Stress resultant constitutive laws should be verified for all possible loading paths before being employed in practical applications. However, it is impossible to examine all possible complex loading conditions. Therefore, uniaxial combined tension and bending loads have been considered to explore the coupling effects between moments and membrane forces (EIDSHEIM \& LARSEN [1981]). On the other hand, BIENIEK and FUNARO [1976] examined their constitutive law under cyclic uniaxial bending. Also, under uniaxial combined tension and bending conditions, CHOU et al. [1991] examined their constitutive law of deformation plasticity nature in proportional loading and prestretching cases, where the states of the prestretching are below the initial yield state. The results based on the constitutive equation of CHou et al. [1991] are very close to those for the corresponding elastic-plastic materials using the through-the-thickness integration method.

In this paper, we continue to assess the applicability of the stress resultant theory, which includes the modified Ilyushin yield surface and the hardening rule of CHOU et al. [1993], the associate flow rule, and the finite deformation effects. Preloading and then unloading cases under uniaxial combined tension and bending conditions are considered to show the coupling effects of bending and stretching, where the preloadings are beyond the initial yield state.

First, the rate form of the stress resultant constitutive law are derived using the hardening rule and yield surfaces in the stress resultant space of CHOU et al. [1993], which were derived and constructed from the stress resultant constitutive law of CHou et al. [1991] for power-law materials under proportional straining conditions. Then, we reduce the rate form of the stress resultant constitutive law for prestretching and prebending cases under both plane stress and plane strain conditions. Also, for completeness, the incremental stress-strain equations for Mises materials will be presented under both plane stress and plane strain conditions. The results of this through-the-thickness integration method, where the stress resultants are obtained from integrating the stresses through the thickness, will be used as the benchmarks to critically assess the applicability of the stress resultant theory. Furthermore, the through-the-thickness integration method based on the finite deformation approach will also be presented, and its results will be compared with those of the stress resultant theory. This is because the stress resultant theory is applied to the simulation of sheet metal forming processes (CHOU et al. [1994]), where finite deformation effects must be taken account for.

Next, the plastic (residual) midplane strain and curvature will be examined by unloading a plate element from a loading state until the free stress resultant state is reached. During the loading process, prestretching and prebending paths will be chosen. In the prestretching cases, the bending moment increases at a fixed preloaded membrane force; in the prebending cases, the membrane force increases at a fixed preloaded bending moment. In each case, the normalized equivalent stress resultants and normalized equivalent plastic generalized strains will be calculated under both plane stress and plane strain conditions for different hardening exponents. These results will be compared with those 
using the through-the-thickness integration method based on both the small-strain and the finite deformation approaches. The implications of these results will be discussed.

Finally, according to the definition of the yield surface in the stress resultant space, the plastic (residual) midplane strain and curvature remain unchanged and path independent during unloading processes. However, it has not been investigated whether the results of the through-the-thickness integration method are significantly path dependent or independent in the stress resultant space because there may be reverse plastic loading in some layers locally during unloading processes. From this viewpoint, we will investigate the residual midplane strains and curvatures and the local stress distributions under different unloading paths. These unloading paths are as follows: two follow the original loading path in the reverse direction and another follows the proportional unloading path, where the ratio of the bending moment to the membrane force is kept as a constant. The results of this investigation will also be presented and discussed.

\section{A STRESS RESULTANT CONSTITUTIVE LAW}

In this section, a rate form of the stress resultant constitutive law will be constructed with the associate flow rule. Although CHOU et al. [1991] derived a stress resultant constitutive law for a plate element of power-law hardening materials under proportional loading conditions within the context of the small-strain theory, loading paths are usually not guaranteed to be proportional in engineering applications. In general, a rate form of the constitutive relation is employed in elastic-plastic structural analyses because elastic-plastic deformation depends upon prior deformation history.

In the stress resultant theory, a plate element, shown in Fig. 1, is subject to the stress resultant vector $\mathbf{S}$, which is defined as

$$
\mathrm{S}=\left\{N_{11}, N_{22}, N_{12}, M_{11}, M_{22}, M_{12}\right\},
$$

where $N_{\alpha \beta}$ are the membrane forces and $M_{\alpha \beta}$ are the bending moments. In this paper, Greek subscripts range from 1 to 2 . The local coordinate system $x_{1}, x_{2}$, and $x_{3}$ for the plate element is fixed at the reference surface with the $x_{1}$ and $x_{2}$ axes being tangent to the surface. The reference surface is the middle surface of the plate element within the context of the small-strain approach. When the finite deformation of the plate element is considered due to the change of thickness, the reference surface is the deformed con-

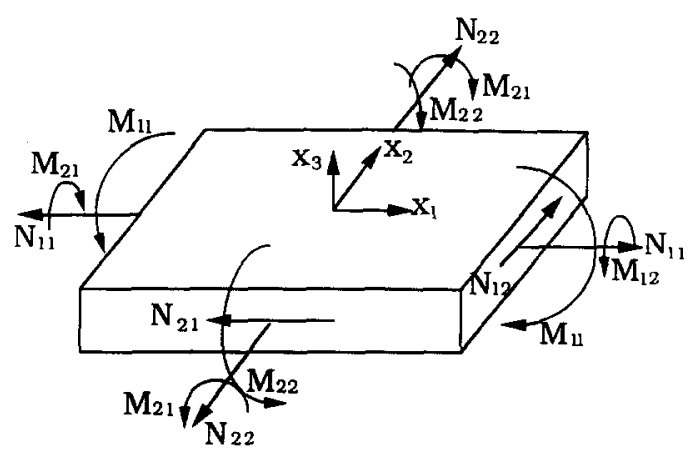

Fig. 1. Conventions of a plate element. 
figuration of the middle surface in the undeformed configuration. The work-conjugate generalized strain vector $\mathbf{E}$ is defined as

$$
\mathbf{E}=\left\{e_{11}, e_{22}, 2 e_{12}, \kappa_{11}, \kappa_{22}, 2 \kappa_{12}\right\},
$$

where $e_{\alpha \beta}$ are the midplane strains and $\kappa_{\alpha \beta}$ are the curvatures.

The components of the generalized strain rate $\dot{E}_{i}$ are assumed to be decomposed into elastic parts $\dot{E}_{i}^{e}$ and plastic parts $\dot{E}_{i}^{p}$

$$
\dot{E}_{i}=\dot{E}_{i}^{e}+\dot{E}_{i}^{p}
$$

where the subscript $i$ has a range from 1 to 6 . It should be mentioned that the additive decomposition in eqn (1) is generally assumed in the classical small-strain plasticity theory. We assume this additive decomposition in eqn (1) in order to maintain the simplicity and practical applicability of the theory for the range of the strains typically encountered in sheet metal forming applications (CHOU et al. [1994]). The components of the plastic generalized strain rate $\dot{E}_{i}^{p}$ are defined, analogous to the associate flow rule of the classical plasticity theory, as

$$
\dot{E}_{i}^{p}=\dot{\Lambda} \frac{\partial F}{\partial S_{i}}
$$

where $\dot{A}$ is a proportionality and $F$ is the yield function. The yield function is assumed to be a linear function of normalized stress resultant invariants (CHOU et al. [1993])

$$
F=\left\{I_{N}+\mu I_{M}+\bar{\omega}\left|I_{M N}\right|\right\}^{1 / 2}=\bar{\Sigma},
$$

where the parameters $\mu$ and $\bar{\omega}$ depend upon the power-law hardening exponent $n$ of the stress-strain relation in a tensile test. In eqn (3), $\bar{\Sigma}$ represents the normalized equivalent stress resultant, and $I_{N} I_{M}$, and $I_{M N}$ are the second order invariants of the normalized stress resultants, $\bar{N}_{\alpha \beta}$ and $\bar{M}_{\alpha \beta}$, as follows:

$$
\begin{aligned}
& I_{N}=\bar{N}_{11}^{2}+\bar{N}_{22}^{2}-\bar{N}_{11} \bar{N}_{22}+3 \bar{N}_{12}^{2}, \\
& I_{M}=\bar{M}_{11}^{2}+\bar{M}_{22}^{2}-\bar{M}_{11} \bar{M}_{22}+3 \bar{M}_{12}^{2},
\end{aligned}
$$

and

$$
I_{M N}=\bar{N}_{11} \bar{M}_{11}+\bar{N}_{22} \bar{M}_{22}-\frac{1}{2} \bar{N}_{11} \bar{M}_{22}-\frac{1}{2} \bar{N}_{22} \bar{M}_{11}+3 \bar{N}_{12} \bar{M}_{12}
$$

where $\bar{N}_{\alpha \beta}=N_{\alpha \beta} / \sigma_{0} h$, and $\bar{M}_{\alpha \beta}=4 M_{\alpha \beta} / \sigma_{0} h^{2}$. Here, $\sigma_{0}$ is taken as the initial yield stress of the tensile test, and $h$ is the current plate thickness when finite deformation effects are considered. Within the small-strain approach, however, $h$ is just assumed to be the initial plate thickness.

The stress resultant rate $\mathbf{S}$ and the elastic generalized strain rate $\dot{\mathbf{E}}^{\mathbf{e}}$ are assumed to be related via the usual elastic modulus $\mathbf{D}^{\mathbf{e}}$ as

$$
\dot{S}_{i}=D_{i j}^{e} \dot{E}_{j}^{e}=D_{i j}^{e}\left(\dot{E}_{j}-\dot{E}_{j}^{p}\right),
$$


where the subscripts $i$ and $j$ have a range from 1 to 6 . The components of the elastic modulus $\mathbf{D}^{\mathbf{e}}$ are

$$
\left[\mathbf{D}^{\mathbf{e}}\right]=\frac{E h}{1-\nu^{2}}\left(\begin{array}{cccccc}
1 & \nu & 0 & 0 & 0 & 0 \\
\nu & 1 & 0 & 0 & 0 & 0 \\
0 & 0 & \frac{1-\nu}{2} & 0 & 0 & 0 \\
0 & 0 & 0 & \frac{h^{2}}{12} & \frac{\nu h^{2}}{12} & 0 \\
0 & 0 & 0 & \frac{\nu h^{2}}{12} & \frac{h^{2}}{12} & 0 \\
0 & 0 & 0 & 0 & 0 & \frac{(1-\nu) h^{2}}{24}
\end{array}\right) \text {, }
$$

where $E$ is Young's modulus and $\nu$ is Poisson's ratio.

The consistency condition dictates the stress resultant state during plastic flow to remain on the yield surface in the stress resultant space. It is

$$
\frac{\partial F}{\partial S_{i}} \dot{S}_{i}+\frac{\partial F}{\partial h} \dot{h}=\frac{\partial \bar{\Sigma}}{\partial \Lambda} \dot{\Lambda},
$$

where $\dot{h}=-\left(\dot{E}_{1}+\dot{E}_{2}\right) h$ is assumed. Multiplying eqn (4) by $\partial F / \partial S_{i}$ and combining with eqns (2) and (5) give the proportionality of plastic flow rate as

$$
\dot{\Lambda}=\frac{\frac{\partial F}{\partial S_{m}} D_{m n}^{e} \dot{E}_{n}-\frac{\partial F}{\partial h}\left(\dot{E}_{1}+\dot{E}_{2}\right) h}{\frac{\partial \bar{\Sigma}}{\partial \Lambda}+\frac{\partial F}{\partial S_{k}} D_{k l}^{e} \frac{\partial F}{\partial S_{l}}}
$$

where the subscripts $k, l, m$, and $n$ have a range from 1 to 6 .

The rate form of the stress resultant constitutive law is derived by substituting eqn (6) into eqns (2) and (4) as

$$
\dot{S}_{i}=D_{i j}^{e p} \dot{E}_{j}
$$

The tangent moduli $D_{i j}^{e p}$ in eqn (7) are derived as

$$
D_{i j}^{e p}=D_{i k}^{e}\left(I_{k j}-\frac{\frac{\partial F}{\partial S_{k}}\left(\frac{\partial F}{\partial S_{l}} D_{l j}^{e}-\frac{\partial F}{\partial h}\left(\delta_{1 j}+\delta_{2 j}\right) h\right)}{\frac{\partial \bar{\Sigma}}{\partial \Lambda}+\frac{\partial F}{\partial S_{m}} D_{m n}^{e} \frac{\partial F}{\partial S_{n}}}\right),
$$

where $I_{i j}$ are the elements of the unit matrix. Also, $\partial F / \partial S_{i}$ and $\partial F / \partial h$ can be derived from the yield function in eqn (3) as 


$$
\begin{aligned}
& \frac{\partial F}{\partial S_{1}}=\frac{1}{\sigma_{0}^{2} \bar{\Sigma}}\left\{\frac{1}{h^{2}}\left(2 N_{11}-N_{22}\right)+\frac{4 \bar{\omega} \lambda}{h^{3}}\left(M_{11}-\frac{M_{22}}{2}\right)\right\}, \\
& \frac{\partial F}{\partial S_{2}}=\frac{1}{\sigma_{0}^{2} \bar{\Sigma}}\left\{\frac{1}{h^{2}}\left(2 N_{22}-N_{11}\right)+\frac{4 \bar{\omega} \lambda}{h^{3}}\left(M_{22}-\frac{M_{11}}{2}\right)\right\}, \\
& \frac{\partial F}{\partial S_{3}}=\frac{1}{\sigma_{0}^{2} \overline{\bar{\Sigma}}}\left\{\frac{6 N_{12}}{h^{2}}+\frac{12 \bar{\omega} \lambda M_{12}}{h^{3}}\right\}, \\
& \frac{\partial F}{\partial S_{4}}=\frac{1}{\sigma_{0}^{2} \bar{\Sigma}}\left\{\frac{16 \mu}{h^{4}}\left(2 M_{11}-M_{22}\right)+\frac{4 \bar{\omega} \lambda}{h^{3}}\left(N_{11}-\frac{N_{22}}{2}\right)\right\}, \\
& \frac{\partial F}{\partial S_{5}}=\frac{1}{\sigma_{0}^{2} \bar{\Sigma}}\left\{\frac{16 \mu}{h^{4}}\left(2 M_{22}-M_{11}\right)+\frac{4 \bar{\omega} \lambda}{h^{3}}\left(N_{22}-\frac{N_{11}}{2}\right)\right\}, \\
& \left.\frac{\partial F}{\partial S_{6}}=\frac{1}{\sigma_{0}^{2} \overline{\bar{\Sigma}}}\left\{\frac{96 \mu M_{12}}{h^{4}}+\frac{12 \bar{\omega} \lambda N_{12}}{h^{3}}\right)\right\},
\end{aligned}
$$

and

$$
\frac{\partial F}{\partial h}=-\frac{1}{\bar{\Sigma}}\left\{\frac{2}{h} I_{N}+\mu \frac{4}{h} I_{M}+\bar{\omega} \frac{3}{h}\left|I_{M N}\right|\right\},
$$

where $\lambda=\operatorname{sgn}\left(I_{M N}\right)\left(\lambda=1\right.$ when $I_{M N}>0$ and $\lambda=-1$ when $\left.I_{M N}<0\right)$.

To determine the value of $\partial \bar{\Sigma} / \partial \Lambda$, the hardening rule for power-law materials in CHOU et al. [1993] based on the small-strain approach is adopted as

$$
\bar{\Sigma}=\eta \bar{\Lambda}^{1 / n},
$$

where $n$ is the power-law hardening exponent, and $\eta$ is a constant dependent on the deformation state. The value of $\eta$ is very close to 1 and the maximum deviation from 1 is $4 \%$. Therefore, $\eta$ is assumed to be 1 for simplicity. Here, $\bar{\Lambda}$ is the normalized equivalent plastic generalized strain accumulated through the deformation history as

$$
\bar{\Lambda}=\int_{0}^{t} \dot{\bar{\Lambda}} d t
$$

The relation between the normalized equivalent plastic generalized strain rate $\dot{\bar{A}}$ and the proportionality $\dot{A}$ is

$$
\dot{\bar{\Lambda}}=\dot{\Lambda} E / \sigma_{0}^{2} h
$$

The normalized equivalent plastic generalized strain rate can be described (CHou et al. [1993]) as

$$
\dot{\bar{\Lambda}}=\left[\frac{4}{3}\left(I_{\dot{e}}+\frac{9}{16 \mu} I_{\dot{\kappa}}-\frac{3 \bar{\omega} \lambda}{8 \mu} I_{\dot{e} \dot{\kappa}}\right)\right]^{1 / 2}
$$


Here, $I_{\dot{e}}, I_{\dot{\kappa}}$, and $I_{\dot{e} \dot{k}}$ are the second order invariants of the normalized plastic generalized strain rates as follows:

$$
\begin{aligned}
& I_{\dot{e}}=\dot{\bar{e}}_{11}^{p 2}+\dot{\bar{e}}_{22}^{p^{2}}+\dot{\bar{e}}_{11}^{p} \dot{\bar{e}}_{22}^{p}+\dot{\bar{e}}_{12}^{p 2}, \\
& I_{\dot{\kappa}}=\dot{\bar{\kappa}}_{11}^{p 2}+\dot{\bar{\kappa}}_{22}^{p 2}+\dot{\bar{\kappa}}_{11}^{p} \dot{\bar{\kappa}}_{22}^{p}+\dot{\bar{\kappa}}_{12}^{p 2},
\end{aligned}
$$

and

$$
I_{\dot{e} \dot{\kappa}}=2 \dot{\bar{e}}_{11}^{p} \dot{\bar{\kappa}}_{11}^{p}+2 \dot{\bar{e}}_{22}^{p} \dot{\bar{\kappa}}_{22}^{p}+\dot{\bar{e}}_{22}^{p} \dot{\bar{\kappa}}_{11}^{p}+\dot{\bar{e}}_{11}^{p} \dot{\bar{\kappa}}_{22}^{p}+2 \dot{\bar{e}}_{12}^{p} \dot{\bar{\kappa}}_{12}^{p}
$$

where $\dot{\bar{e}}_{\alpha \beta}=\dot{e}_{\alpha \beta} E / \sigma_{0}$ and $\dot{\bar{\kappa}}_{\alpha \beta}=\dot{\kappa}_{\alpha \beta} E h / 3 \sigma_{0}$. From eqns (9) and (11), $\partial \bar{\Sigma} / \partial \Lambda$ can be written as

$$
\frac{\partial \bar{\Sigma}}{\partial \Lambda}=\frac{\partial \bar{\Sigma}}{\partial \bar{\Lambda}} \frac{\partial \bar{\Lambda}}{\partial \Lambda}=\frac{E}{n \sigma_{0}^{2} h} \bar{\Sigma}^{1-n} .
$$

Note that when the stress resultant theory is applied to analyses with the small-strain assumption, the undeformed geometry can be treated as the reference configuration according to the small-strain theory. Then, $h$ is treated as a constant in the normalized terms and the terms related to the thickness change in eqn (5) can be eliminated.

It should be noted that it is impossible to benchmark all the possible loading paths for the stress resultant theory. In this paper, we will examine a limited number of uniaxial combined bending and membrane force cases under plane stress and plane strain conditions as many previous studies on stress resultant theories (CRISFIELD [1974], BIENIEK \& FUNARO [1976], EIDSHEIM \& LARSEN [1981]). Then, eqn (8) can be simplified for these special cases. Under plane stress conditions, the conditions of $\dot{\bar{\epsilon}}_{22}^{p}=-\dot{\bar{e}}_{11}^{p} / 2$ (due to incompressibility) and $\dot{\bar{\epsilon}}_{12}^{p}=0$ lead to $\dot{\bar{e}}_{22}^{p}=-\dot{\bar{e}}_{11}^{p} / 2, \dot{\bar{\kappa}}_{22}^{p}=-\dot{\bar{\kappa}}_{11}^{p} / 2, \dot{\bar{e}}_{12}^{p}=0$, and $\dot{\bar{\kappa}}_{12}^{p}=0$. The normalized equivalent plastic generalized strain rate is

$$
\dot{\bar{\Lambda}}=\left(\dot{\bar{e}}_{11}^{p 2}+\frac{9}{16 \mu} \dot{\bar{\kappa}}_{11}^{p 2}-\frac{3 \bar{\omega} \lambda}{8 \mu} \dot{\bar{e}}_{11}^{p} \dot{\bar{\kappa}}_{11}^{p}\right)^{1 / 2} \text {. }
$$

Moreover, $\partial F / \partial S_{2}=-\frac{1}{2}\left(\partial F / \partial S_{1}\right)$ and $\partial F / \partial S_{5}=-\frac{1}{2}\left(\partial F / \partial S_{4}\right)$. The lateral deformation measures $e_{22}$ and $\kappa_{22}$ are functions of $e_{11}$ and $\kappa_{11}$ because $N_{22}=M_{22}=0$. Then, the relations between $N_{11}, M_{11}$ and $e_{11}, \kappa_{11}$ can be established from eqn (8). Under plane strain conditions, the normalized equivalent plastic generalized strain rate can be simplified as

$$
\dot{\bar{\Lambda}}=\left[\frac{4}{3}\left(\dot{\bar{e}}_{11}^{p 2}+\frac{9}{16 \mu} \dot{\bar{\kappa}}_{11}^{p 2}-\frac{3 \bar{\omega} \lambda}{8 \mu} \dot{\bar{e}}_{11}^{p} \dot{\bar{\kappa}}_{11}^{p}\right)\right]^{1 / 2} \text {. }
$$

With the constraint $e_{11}=0$ and $\kappa_{11}=0$, the relations between $N_{11}, M_{11}$, and $e_{11}, \kappa_{11}$ can be easily established from eqn (8) directly.

\section{THE THROUGH-THE-THICKNESS INTEGRATION METHOD}

To benchmark the constitutive behavior of the stress resultant theory, we investigate the plate element constitutive response using the corresponding elastic-plastic stressstrain equations for each layer of material through the thickness and calculate stress 
resultants by integrating the stress through the thickness. The plastic hardening behavior for the corresponding Mises materials is taken as

$$
\frac{\epsilon^{p}}{\epsilon_{0}}=\left(\frac{\sigma_{e}}{\sigma_{0}}\right)^{n}-1,
$$

where $\epsilon_{0}=\sigma_{0} / E, \sigma_{e}\left(=\left(\frac{2}{3} s_{i j} s_{i j}\right)^{1 / 2}\right.$, where $\left.s_{i j}=\sigma_{i j}-\frac{1}{3} \sigma_{k k} \delta_{i j}\right)$ is the effective stress, and $\epsilon^{p}$ is the effective plastic strain. The effective plastic strain is obtained from integration over the deformation history based on the effective plastic strain increment $d \epsilon^{p}$ [ $=\left(\frac{2}{3} d \epsilon_{i j}^{p} d \epsilon_{i j}^{p}\right)^{1 / 2}$, where $d \epsilon_{i j}^{p}$ are the plastic strain increments].

Now, the constitutive behavior of elastic-plastic materials is discussed for each layer of the thin plate element where $\sigma_{3 i}=0(i=1,2,3)$ (see Fig. 1). Under plane stress conditions (simple beam problem, $\sigma_{2 i}=0, i=1,2,3$ ), the incremental elastic stress-strain relation is

$$
d \sigma_{11}=E d \epsilon_{11}
$$

The incremental stress-strain relation under plastic loading is

$$
d \sigma_{11}=\frac{E H^{\prime}}{E+H^{\prime}} d \epsilon_{11}
$$

where $H^{\prime}=d \sigma_{e} / d \epsilon^{p}$. Under plane strain conditions $\left(\epsilon_{2 i}=0, i=1,2,3\right)$, the incremental elastic relations are

$$
d \sigma_{11}=\frac{E}{1-\nu^{2}} d \epsilon_{11}
$$

and

$$
d \sigma_{22}=\frac{E \nu}{1-\nu^{2}} d \epsilon_{11}
$$

The incremental relations under plastic loading are

$$
d \sigma_{11}=\frac{\left[2 H^{\prime} \sigma_{e}^{2}+\frac{E}{2}\left(\sigma_{11}-2 \sigma_{22}\right)^{2}\right]}{2\left(1-\nu^{2}\right) \frac{H^{\prime}}{E} \sigma_{e}^{2}+\left[\left(\frac{5}{2}-2 \nu\right) \sigma_{11}^{2}+(5 \nu-4) \sigma_{11} \sigma_{22}+\left(\frac{5}{2}-2 \nu\right) \sigma_{22}^{2}\right]} d \epsilon_{11}
$$

and

$$
d \sigma_{22}=\frac{\left[2 \nu H^{\prime} \sigma_{e}^{2}-\frac{E}{2}\left(2 \sigma_{11}-\sigma_{22}\right)\left(2 \sigma_{22}-\sigma_{11}\right)\right]}{2\left(1-\nu^{2}\right) \frac{H^{\prime}}{E} \sigma_{e}^{2}+\left[\left(\frac{5}{2}-2 \nu\right) \sigma_{11}^{2}+(5 \nu-4) \sigma_{11} \sigma_{22}+\left(\frac{5}{2}-2 \nu\right) \sigma_{22}^{2}\right]} d \epsilon_{11}
$$


Next, we consider the through-the-thickness integration method based on the smallstrain approach and the finite deformation approach. Within the small-strain approach, the current configuration is assumed to be the same as the initial configuration. Therefore, the thickness of plate element is assumed not to change during deformation. The strain increments, according to the Kirchhoff assumption, are

$$
d \epsilon_{11}=d e_{11}+z d \kappa_{11}, \quad d \epsilon_{22}=d e_{22}+z d \kappa_{22} .
$$

The membrane forces and bending moments are obtained from integrating stresses through the thickness as follows:

$$
N_{11}=\int_{-h / 2}^{h / 2} \sigma_{11} d z, \quad N_{22}=\int_{-h / 2}^{h / 2} \sigma_{22} d z
$$

and

$$
M_{11}=\int_{-h / 2}^{h / 2} \sigma_{11} z d z, \quad M_{22}=\int_{-h / 2}^{h / 2} \sigma_{22} z d z,
$$

where $h$ is the initial thickness and the stresses are accumulated from the increments of the stresses in eqns (17)-(22). In general, numerical methods are needed for the integration through the thickness to obtain the resultants in eqns (24) and (25) when the relations between the stresses and strains are not linear. Here, we subdivided the interval of integration into 50 equal subintervals, and consequently, we used a simple rectangular rule for the integration.

Within the finite deformation approach, the current thickness is different from the initial thickness. The thickness of each layer $\delta h$ should be updated according to

$$
d \epsilon_{33}=\frac{d \delta h}{\delta h},
$$

where $d \epsilon_{33}$ is the out-of-plane strain increment, and $d \delta h$ is the thickness increment for the current layer. Under plane stress conditions, the strain increment of each layer is

$$
d \epsilon_{33}=-\frac{2 \nu \frac{H^{\prime}}{E}+1}{2\left(\frac{H^{\prime}}{E}+1\right)} d \epsilon_{11}
$$

and under plane strain conditions, the strain increment of each layer is

$$
d \epsilon_{33}=-\frac{2 \nu(1+\nu) \frac{H^{\prime}}{E} \sigma_{e}^{2}+\left[(1+\nu) \sigma_{11}^{2}+\left(\frac{1}{2}-4 \nu\right) \sigma_{11} \sigma_{22}+\left(4 \nu-\frac{1}{2}\right) \sigma_{22}^{2}\right]}{2\left(1-\nu^{2}\right) \frac{H^{\prime}}{E} \sigma_{e}^{2}+\left[\left(\frac{5}{2}-2 \nu\right) \sigma_{11}^{2}+(5 \nu-4) \sigma_{11} \sigma_{22}+\left(\frac{5}{2}-2 \nu\right) \sigma_{22}^{2}\right]} d \epsilon_{1} .
$$


The true strain definition is adopted in this approach as follows:

$$
\epsilon_{11}=\ln \lambda_{1}, \quad \epsilon_{22}=\ln \lambda_{2}
$$

Here, $\lambda_{1}$ and $\lambda_{2}$ are the stretch ratios in the $x_{1}$ and $x_{2}$ directions. According to the Kirchhoff assumption, they can be written as:

$$
\lambda_{1}=\lambda_{1}^{0}\left(1+z \kappa_{11}\right), \quad \lambda_{2}=\lambda_{2}^{0}\left(1+z \kappa_{22}\right),
$$

where $\lambda_{1}^{0}$ and $\lambda_{2}^{0}$ are the stretch ratios of the reference surface in the $x_{1}$ and $x_{2}$ directions. Consequently, the strain rates are

$$
\dot{\epsilon}_{11}=\dot{e}_{11}+\frac{z \dot{\kappa}_{11}+\kappa_{11} \dot{z}}{1+\kappa_{11} z}, \quad \dot{\epsilon}_{22}=\dot{e}_{22}+\frac{z \dot{\kappa}_{22}+\kappa_{22} \dot{z}}{1+\kappa_{22} z}
$$

where $\dot{e}_{11}=\dot{\lambda}_{1}^{0}$ and $\dot{e}_{22}=\dot{\lambda}_{2}^{0}$. The membrane forces and bending moments are obtained from integrating stresses through the thickness as follows:

$$
N_{11}=\int_{h} \sigma_{11} d z, \quad N_{22}=\int_{h} \sigma_{22} d z
$$

and

$$
M_{11}=\int_{h} \sigma_{11} z d z, \quad M_{22}=\int_{h} \sigma_{22} z d z
$$

where $\sigma_{11}$ and $\sigma_{22}$ are the Cauchy stresses. These integrations are performed through the current thickness $h$.

Note that under plane strain conditions, $\dot{\epsilon}_{22}=\dot{\kappa}_{22}=0$, and under plane stress conditions, $N_{22}=M_{22}=0$. Finally, the normalized equivalent stress resultant is calculated from eqn (3), and the normalized equivalent plastic generalized strain rate is calculated from eqns (10) and (14) under plane stress conditions and from eqns (10) and (15) under plane strain conditions.

\section{A COMPARISON BETWEEN THE RESULTS OF THE STRESS RESULTANT THEORY AND THE THROUGH-THE-THICKNESS INTEGRATION METHOD}

In this section, we will investigate the relation between the normalized equivalent stress resultant $\bar{\Sigma}$ and the normalized equivalent plastic generalized strain $\bar{\Lambda}$, using both the stress resultant theory and the through-the-thickness integration method. The plastic (residual) generalized strains for preloading cases are determined after unloading a plate element from any loading state until the free stress resultant state is reached. According to the associate flow rule of the isotropic hardening model in the stress resultant space, no plastic part of the generalized strain increment is generated during this unloading process. However, the stress resultants are determined using integration from the local stresses of each layer when the through-the-thickness integration method is used. Although the plate element is under unloading conditions in the stress resultant space, each layer of the plate element has three possible loading types in the stress space: plas- 
tic loading, elastic unloading, or reverse plastic loading. The loading type for each layer is dependent upon the unloading path in the stress resultant space. Consequently, there may be plastic strains generated locally in some layers during this unloading process. Therefore, the plastic generalized stains are globally defined from the residual generalized strains at the free stress resultant state when the through-the-thickness integration method is used.

To understand the effect of the coupling of moments and membrane forces, we investigate cases under combined membrane force and bending moment. We control the magnitudes of $N_{11}$ and $M_{11}$ to follow the loading and unloading paths (regardless of $N_{22}$ and $M_{22}$ under plane strain conditions) as shown in Fig. 2. The loading paths include prestretching cases (path $\boldsymbol{P}_{s}$ ) and prebending cases (path $\boldsymbol{P}_{b}$ ). In the prestretching cases, the bending moment $M_{11}$ increases at a fixed preloaded membrane force $N_{11}$; in the prebending cases, the membrane force $N_{11}$ increases at a fixed preloaded bending moment $M_{11}$. The unloading paths are those following the original loading path (path $P_{s}$ or $P_{b}$ ) in the reverse direction (path EP-1 or EP-2).

When the through-the-thickness integration method is used, eqns (17)-(22), (24), (25), (32), and (33) determine the plastic generalized strain increments and the stress resultant increments in each incremental step. Then, the normalized equivalent stress resultants are determined from eqn (3) and the normalized equivalent plastic generalized strain increments from eqns (14) and (15). When the stress resultant theory is used, the plastic generalized strain increments are determined from eqn (9) for a given stress resultant increment. Also, eqns (2), (3), and (6) determine the yield surface and the plastic generalized strain at the preloading.

Based on the small-strain approach where the thickness of the element is assumed not to change as a function of deformation, the results under plane stress (simple beam) conditions are shown in Figs. 3 and 4, and the results under plane strain conditions are shown in Figs. 5 and 6. In these figures, the curves marked as "Power Law" represent the results based on the stress resultant theory with the power-law hardening rule, and the curves marked as "Through-the-Thickness Integration" represent the results based on the through-the-thickness integration method. In these figures, $N_{\text {pre }}$ represents the preloaded membrane force normalized by $N_{L}\left(=\sigma_{0} h\right)$ and $M_{\text {pre }}$ represents the preloaded bending moment normalized by $M_{L}\left(=\sigma_{0} h^{2} / 4\right)$.

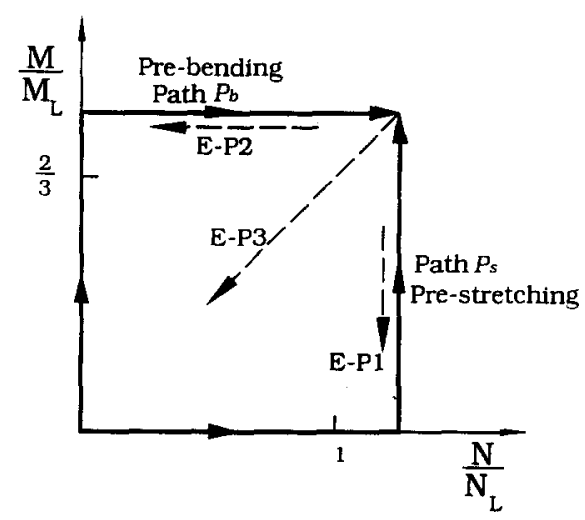

Fig. 2. Loading and unloading paths in the stress resultant space for prestretching, prebending, and unloading cases. 


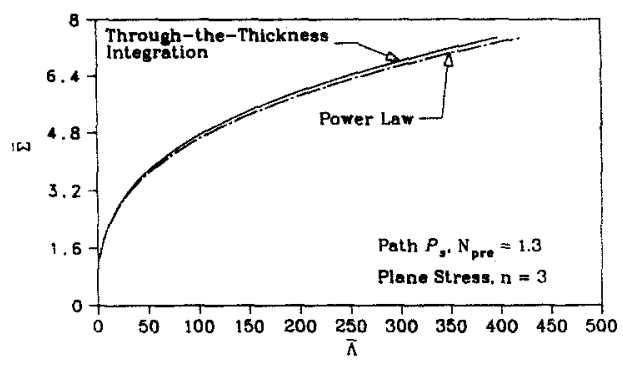

(a)

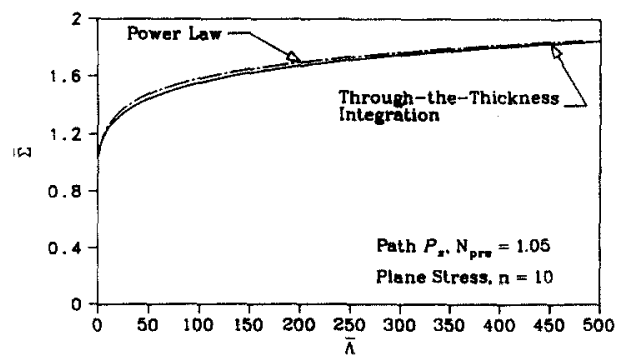

(b)

Fig. 3. Comparison of the relation between the normalized equivalent stress resultant and the normalized equivalent plastic generalized strain based on the small-strain approach under plane stress conditions along loading path $P_{s}$. (a) $n=3$. (b) $n=10$.

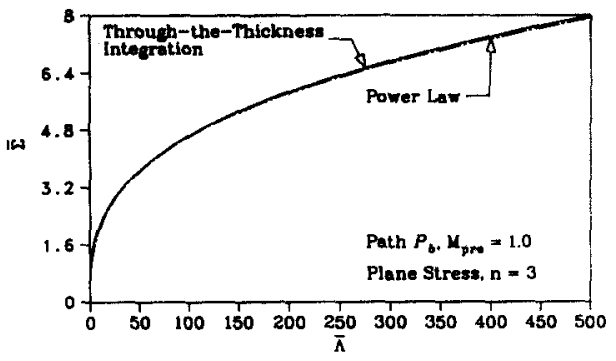

(a)

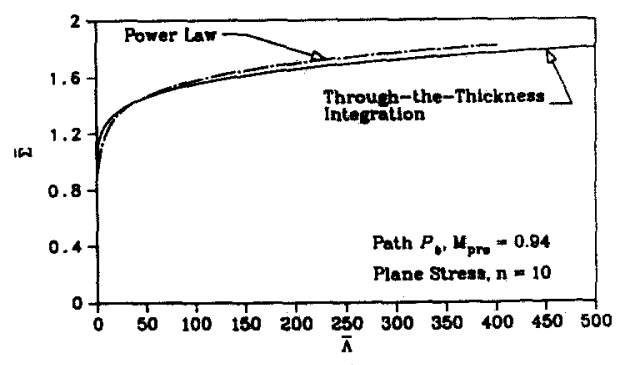

(b)

Fig. 4. Comparison of the relation between the normalized equivalent stress resultant and the normalized equivalent plastic generalized strain based on the small-strain approach under plane stress conditions along loading path $P_{b}$. (a) $n=3$. (b) $n=10$. 


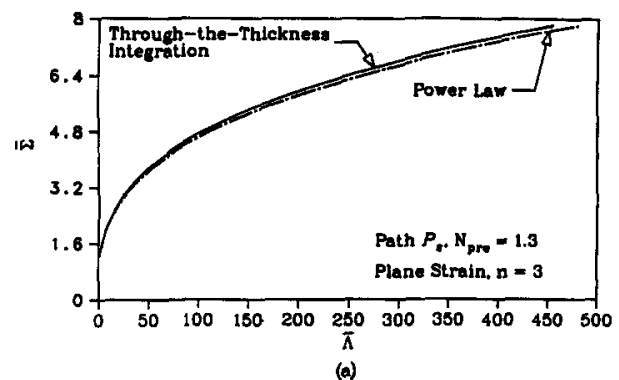

(a)

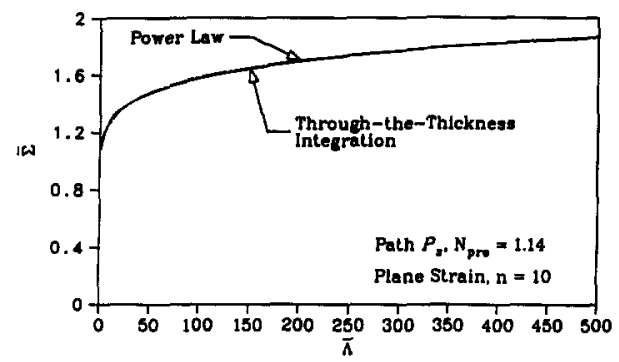

(b)

Fig. 5. Comparison of the relation between the normalized equivalent stress resultant and the normalized equivalent plastic generalized strain based on the small-strain approach under plane strain conditions along loading path $P_{s}$. (a) $n=3$. (b) $n=10$.
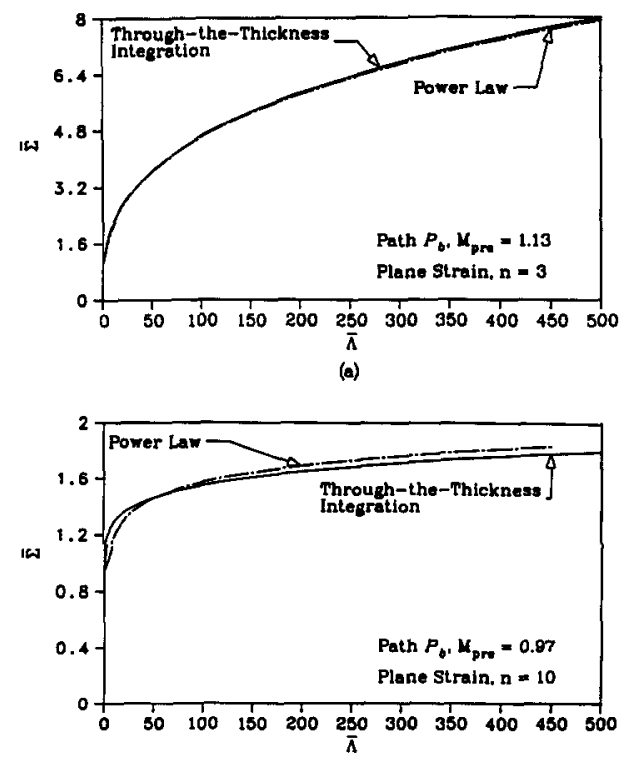

(b)

Fig. 6. Comparison of the relation between the normalized equivalent stress resultant and the normalized equivalent plastic generalized strain based on the small-strain approach under plane strain conditions along loading path $P_{b}$. (a) $n=3$. (b) $n=10$. 
Under plane stress conditions, the results of the normalized equivalent stress resultant $\bar{\Sigma}$ as a function of the normalized equivalent generalized plastic strain $\bar{A}$ along path $P_{s}$ are shown in Fig. 3, and those along path $P_{b}$ are shown in Fig. 4 . In general, the results from both the through-the-thickness integration method and the stress resultant theory agree very well with each other. A detailed comparison shows that the stress resultant theory gives a bit softer results than the through-the-thickness integration method for $n=3$. However, for $n=10$, the stress resultant theory gives a bit softer and then a bit stiffer results than the through-the-thickness integration method. Typically, the $n$ value ranges from 4 to 6.8 for sheet metal used in forming operations. A possible source of the error using the stress resultant theory is the assumption of the value for the constant $\eta$ in the hardening rule in eqn (9). As shown in CHou et al. [1993], the constant $\eta$ can have a deviation of $4 \%$ from 1 under proportional loading conditions. Another possible source of error is the approximation of shape in the modified Ilyushin yield surface in eqn (3) where a quadratic function is chosen to fit the complementary potential surface of CHOU et al. [1991] for simplicity.

Note that there are very small differences of the relations between the normalized equivalent stress resultants and the normalized plastic generalized strains under path $P_{S}$ and path $P_{b}$ based on the through-the-thickness integration method when we compare Figs. 3 and 4 . This is because the local phenomena slightly affect the global results and the results are somewhat dependent on the paths. In general, the results of the stress resultant theory are very close to those of the through-the-thickness integration method even under different loading paths.

Under plane strain conditions for $\nu=0.3$, the results of the normalized equivalent stress resultant $\bar{\Sigma}$ as a function of the normalized equivalent generalized plastic strain $\bar{A}$ along path $P_{s}$ are shown in Fig. 5 , and those along path $P_{b}$ are shown in Fig. 6. A comparison shows the same trends as those under plane stress conditions. For $n=3$, the hardening curves of the through-the-thickness integration method in Figs. 3(a), 4(a), 5 (a), and 6(a) are very close to each other. For $n=10$, the hardening curves of the through-the-thickness integration method in Figs. 3(b), 4(b), 5(b), and 6(b) are also very close to each other. This implies that the normalized equivalent stress resultant $\bar{\Sigma}$ and the normalized equivalent generalized plastic strain $\bar{A}$ derived in CHou et al. [1993] are excellent parameters to characterize the plastic deformation of the plate element, since they are consistent under both plane stress and plane strain conditions and different loading paths. In conclusion, the results of the stress resultant theory agree well with those of the through-the-thickness integration method based on the small-strain approach.

Although the stress resultant theory gives good prediction for $\bar{\Lambda}$ up to 500 (about $\epsilon=$ $25 \%$ for AK-steel), the small-strain approach adopted in our through-the-thickness integration method will become inaccurate when the plastic deformation becomes large. At large deformation, the plate thickness must be updated and the true strain measures should be adopted. Therefore, we have to use the results of the through-the-thickness integration method based on the finite deformation approach as discussed earlier to benchmark the results of the stress resultant theory.

Based on the finite deformation approach, the results of the through-the-thickness integration method are shown in Figs. 7 through 10. In these figures, the same symbols and notations are used as those in Figs. 3 through 6 . Figure 7 shows the results under plane stress conditions along path $P_{s}$, and Fig. 8 shows the results under plane stress conditions along path $P_{b}$. A comparison shows that the stress resultant theory gives 


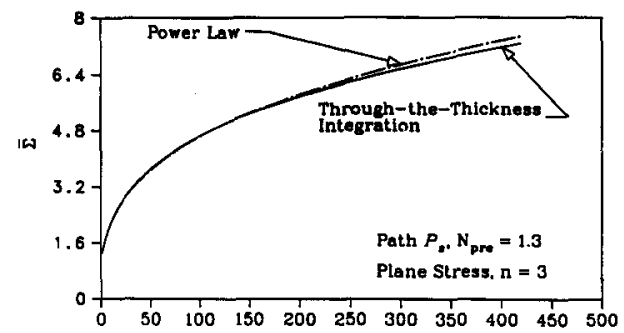

(a)

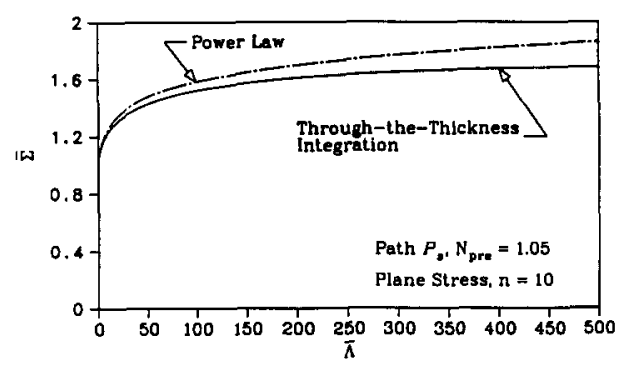

(b)

Fig. 7. Comparison of the relation between the normalized equivalent stress resultant and the normalized equivalent plastic generalized strain based on the finite deformation approach under plane stress conditions along loading path $P_{s}$. (a) $n=3$. (b) $n=10$.

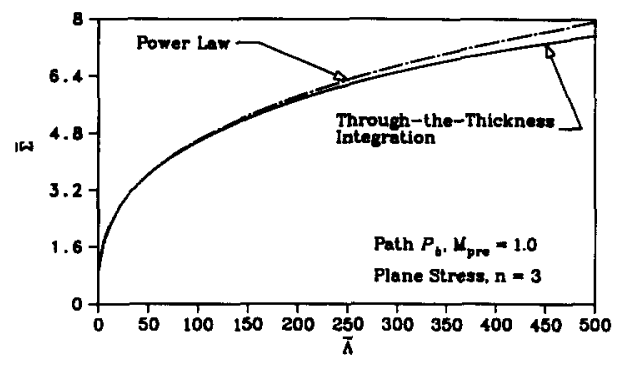

(a)

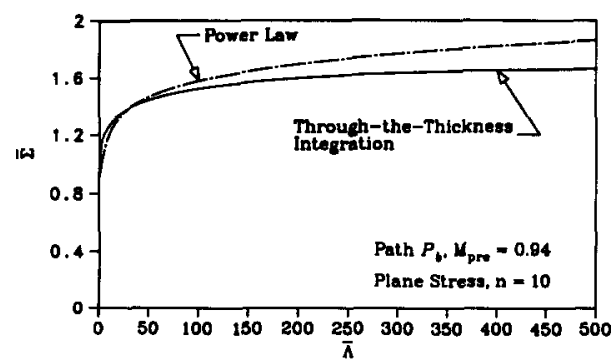

(b)

Fig. 8. Comparison of the relation between the normalized equivalent stress resultant and the normalized equivalent plastic generalized strain based on the finite deformation approach under plane stress conditions along loading path $P_{b}$. (a) $n=3$. (b) $n=10$. 

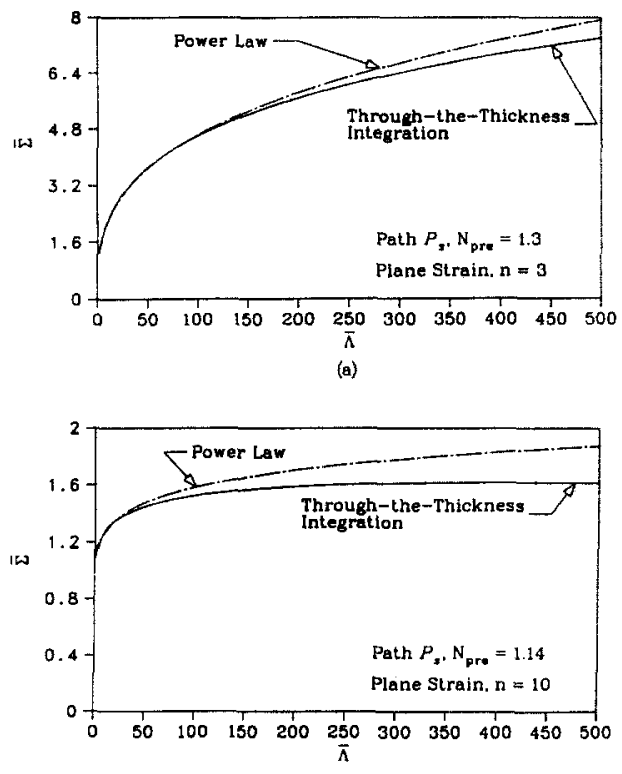

(b)

Fig. 9. Comparison of the relation between the normalized equivalent stress resultant and the normalized equivalent plastic generalized strain based on the finite deformation approach under plane strain conditions along loading path $P_{s}$. (a) $n=3$. (b) $n=10$.

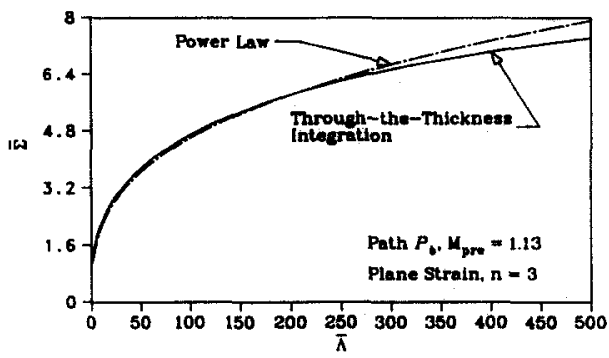

(a)

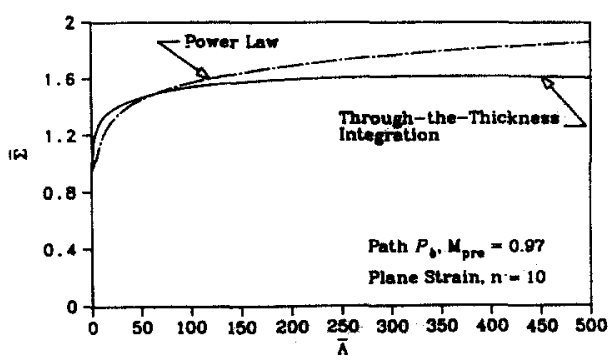

(b)

Fig. 10. Comparison of the relation between the normalized equivalent stress resultant and the normalized equivalent plastic generalized strain based on the finite deformation approach under plane strain conditions along loading path $P_{b}$. (a) $n=3$. (b) $n=10$. 
slightly softer and then stiffer results than those of the through-the-thickness integration method for both $n=3$ and 10 . The differences gradually become large as the normalized equivalent plastic generalized strain increases. This effect becomes more significant as the hardening exponent increases. For high hardening material $(n=3)$, the stress resultant theory gives good approximations when compared with those of the throughthe-thickness integration method. However, for low hardening materials $(n=10)$, an unloading phenomenon appears when the through-the-thickness integration method is used. This unloading cannot be simulated by the stress resultant theory where the hardening rule is described by a monotonically increasing power-law relation. This unloading phenomenon appears when $n>7$ based on a further parametric study. The softening occurs because of the coupling of the moments and the membrane forces and the finite geometry change.

For $\nu=0.3$, Fig. 9 shows the results under plane strain conditions along path $P_{s}$, and Fig. 10 shows the results under plane strain conditions along path $P_{b}$. A comparison of the two figures also shows the same trends as those under plane stress conditions. Unloading phenomenon occurs when $n>7$ as in the plane stress cases. A comparison of the $n=3$ curves and the $n=10$ curves based on the through-the-thickness integration method in Figs. 7 through 10 shows that they all show the same trend as compared with those based on the stress resultant theory. It implies the normalized equivalent stress resultant and the normalized generalized equivalent plastic strain chosen in CHOU et al. [1993] are good scalar parameters to characterize the size of the yield surfaces in the stress resultant space and the corresponding plastic deformation measures even with finite deformation being considered. The results shown in Figs. 7 through 10 suggest the hardening rule should be modified to simulate the softening phenomenon for low hardening materials.

We now investigate the plastic residual strains under different unloading paths using the through-the-thickness integration method. For simplicity, our investigation is restricted to the small-strain approach under plane stress conditions. As shown earlier, the loading paths do not affect the residual plastic strains significantly under the small-strain assumption. We therefore select a plastic deformation state resulted from loading path $P_{s}$ to investigate the unloading path effects on residual plastic strain. We select one unloading path follows the original loading path $P_{s}$ in the reverse direction path shown in Fig. 2 as EP-1. The results are shown by curves UL- $P_{s}$ in Fig. 11. The other unloading path follows the proportional unloading path shown in Fig. 2 as EP-3, where the ratio of the membrane force to the bending moment is kept as a constait. The results are shown by curve UL- $P_{s}$ in Fig. 11. The maximum value of the normalized bending moment is chosen close to the $N_{\text {pre }}$. The results of the two unloading paths for both $n=3$ and $n=10$ are very similar to each other as shown in Fig. 11 . This indicates that the unloading paths do not have a significant effect on the global state variables, i.e. the stress resultants and the generalized plastic strains. This also indicates that the results based on the through-the-thickness integration method are nearly path independent in the stress resultant space for these limited number paths considered here. However, the local stress state which will be illustrated later is greatly affected by the unloading paths. In Fig. 11(a) for $n=3$, it exhibits a discontinuous slope. This is the transition point from preloaded tension to combined tension and bending. In Fig. 11(b) for $n=10$, the discontinuous slope at the transition point cannot be identified easily because of the different scale.

Based on the through-the-thickness integration method, the stress distributions in 


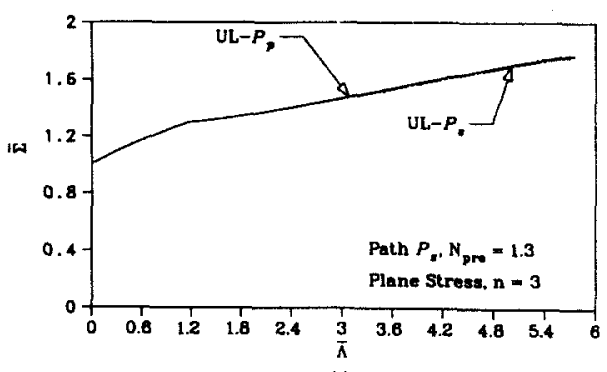

(a)

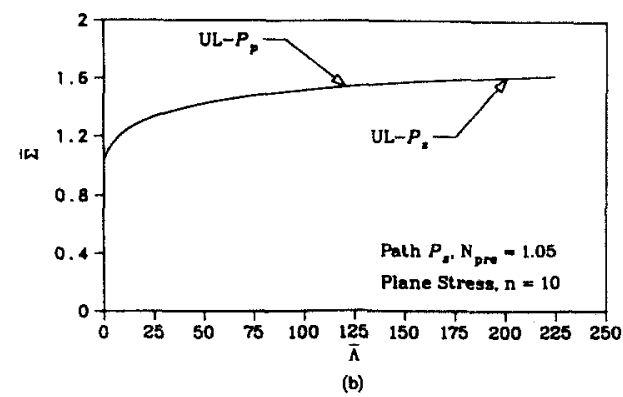

Fig. 11. Comparison of the results based on the through-the-thickness integration method under plane stress conditions along loading path $P_{s}$ and different unloading paths. (a) $n=3$. (b) $n=10$.

the $x_{3}$ direction are shown for different loading and unloading paths in Fig. 12. In Fig. 12(a), curves L- $P_{s}$ and L- $P_{b}$ indicate the stress distributions in the $x_{3}$ direction for paths $P_{s}$ and $P_{b}$, respectively, at $N / N_{L}=M / M_{L}=1.5$ under plane stress conditions. Both stress distributions are quite similar. In Fig. 12(b), curve UL- $P_{s}$ represents the stress distribution after unloading to $N=M=0$ for loading path $P_{s}$ and unloading path E-P1, and curve UL- $P_{b}$ represents the stress distribution after unloading to $N=$ $M=0$ for loading path $P_{b}$ and unloading path E-P2. Since the stress distributions for loading paths $P_{s}$ and $P_{b}$ at $N / N_{0}=M / M_{0}=1.5$ are quite similar, only the results of the stress distributions of curve L- $P_{s}$ after unloading to $N=M=0$ for the proportional unloading path E-P3 are presented by curve UL- $P_{p}$. As shown in the figure, the stress distributions after unloading following the three paths, marked by UL- $P_{s}$, UL- $P_{b}$, and UL- $P_{p}$, are quite different. Note that there is hardly any reverse plastic loading through the thickness for the three unloading paths. This explains the path independence of residual generalized strains based on the through-the-thickness integration method for the unloading paths considered here.

\section{v. CONCLUSION}

It should be noted that an unlimited number of nonproportional loading and unloading paths can be selected for this study. However, we have selected these simple nonproportional loading and unloading paths that represent some typical loading and unloading conditions in sheet forming operations. The results for these paths using the stress resultant theory are compared well with those using the through-the-thickness integration method based on the small-strain approach. However, as suggested by the results 


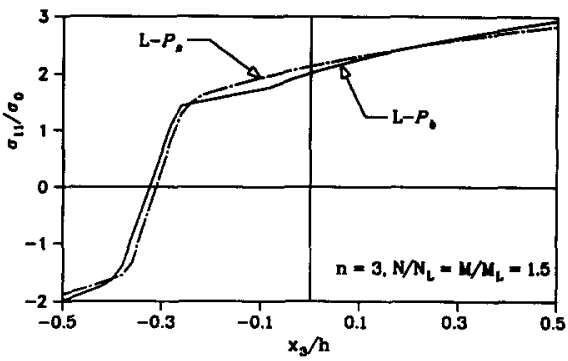

(a)

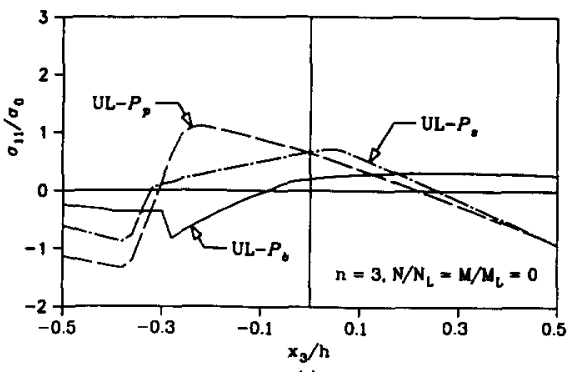

(b)

Fig. 12. (a) The stress distributions (curves L- $P_{s}$ and L- $P_{b}$ ) in the normalized $x_{3}$ direction before unloading at $N / N_{0}=1.5$ and $M / M_{0}=1.5$. (b) The stress distributions (curves UL- $P_{s}$, UL- $P_{b}$, and UL- $P_{p}$ ) in the normalized $x_{3}$ direction after unloading following the reverse directions of the original loading paths and the proportional unloading path.

where finite deformation of the sheet element is considered, the power-law hardening rule for the normalized equivalent stress resultant and the normalized equivalent generalized plastic strain must be modified to take account for the finite deformation effects. A modification of the hardening rule in an ad hoc manner is presented in CHOU et al. [1994] for applications in simulations of sheet metal forming by finite element methods. The finite element simulations based on the stress resultant theory take much less computational time (about $60 \%$ less), and the results are compared well with those based on the through-the-thickness integration method. The investigation presented here forms a part of the theoretical basis for employing the stress resultant theory to simulate sheet metal forming operations.

Acknowledgements - The authors acknowledge the support of this work by the National Science Foundation under Grant Number DDM-9102424 and the University Research Program of the Ford Motor Company.

\section{REFERENCES}

1948 Ilyushr, A.A., Plasticity, Gostekhizdat, Moscow. French translation: Plasticité, Eyrolles, Paris, 1956. 1959 BUdiansKy, B.A., “A Reassessment of Deformation Theories of Plasticity," J. Appl. Mech. (Transactions of the ASME), 26, 259.

1969 Malvern, L.E., Introduction to the Mechanics of a Continuous Medium, Prentice-Hall, Englewood Cliffs, NJ.

1974 CrIsfield, M.A., On an Approximate Yield Criterion for Thin Steel Shells, TRRL Report LR658, Transport and Road Research Laboratory, Crowthorne, Berkshire.

1976 BIENIEK, M.P. and Funaro, J.R., Elasto-Plastic Behavior of Plates and Shells, Report No. DNA 3954T, Weidlinger Associates, New York. 
1978 EgGers, H. and KRöPliN, B., "Yielding of Plates with Hardening and Large Deformations," Int. J. Num. Meth. Engng. 12, 739.

1981 EIDSHEIM, O.M. and LARSEN, P.K., “A Study of Some Generalized Constitutive Methods for Elasto Plastic Shells," in Wunderlich, W., BATHE, K.J., and SteIN, E. (eds.), Nonlinear Finite Element Analysis in Structural Mechanics, Springer, Berlin, pp. 364-384.

1990 Papadopoulos, P. and Taylor, R.L., "Ealsto-Plastic Analysis of Reissner-Mindlin Plates," Appl. Mech. Rev. 43, S40.

1991 Chou, C.H., PAN, J., and TANG, S.C., "Constitutive Laws for Thin Plates of Power-Law Materials," Int. J. Solids Struct. 27, 1387.

1993 Chou, C.H., PAN, J., and TANG, S.C., "A Hardening Rule Between Stress Resultants and Generalized Plastic Strains for Thin Plates of Power-Law Hardening Materials,” J. Appl. Mech. 60, 548.

1994 ChOu, C.H., PAN, J., and TANG, S.C., "Applications of Stress Resultant Constitutive Law to Sheet Metal Forming," Int. J. Numer. Meth. Engng. 37, 717.

Edison Welding Institute

Columbus, $\mathrm{OH}$ 43212, USA

Mechanical Engineering and Applied Mechanics

The University of Michigan

Ann Arbor, MI 48109, USA

Ford Motor Company

Dearborn, MI 48121, USA

(Received 8 July 1991; in final revised form 15 May 1993) 